\title{
Effect of electronic report writing on the quality of nursing report recording
}

\author{
Khadijeh Heidarizadeh ${ }^{1}$, Maryam Rassouli ${ }^{2}$, Houman Manoochehri ${ }^{2}$, Mansoureh Zagheri Tafreshi ${ }^{2}$, Reza Kashef \\ Ghorbanpour $^{3}$
}

${ }^{1} \mathrm{PhD}$ Candidate, School of Nursing \& Midwifery, Shahid Beheshti University of Medical Sciences, Tehran, Iran

${ }^{2} \mathrm{PhD}$, Associate Professor, School of Nursing \& Midwifery, Shahid Beheshti University of Medical Sciences, Tehran, Iran

${ }^{3} \mathrm{PhD}$ Candidate, Iranian Social Security Organization, Tehran, Iran

\section{Type of article: Original}

\begin{abstract}
Background and Aim: Recording performed nursery actions is one of the main chores of nurses. The findings have shown that recorded reports are not qualitatively valid. Since electronic reports can be regarded as a base to write reports, this study aims at determining the effect of utilizing electronic nursing reports on the quality of the records.

Methods: This quasi-experimental study was conducted with the aim of applying an electronic system of nursing recording in the heart department of Shahid Rahimi Medical Center, Lorestan University of Medical Science. The samples were nursing reports on the hospitalized patients in the heart department, the basis of complete enumeration (census) during the fall of 2014. The subjects were sixteen employed nurses. To do the study, the software of nursing records was set based on the Clinical Care Classification system (CCC). The research's tool was the checklist of the Standards of Nursing Documentation.

Results: The findings indicated that before and after the intervention, the amount of reports' adaption with the written standards, respectively, was $(21.8 \%)$ and $(71.3 \%)$, and the most complete recording was medicine status $(58 \%)$ and $(100 \%)$. The worst complete recording before the intervention, acute changes was $(99.1 \%)$ and nursing processes was (78\%) and after, the medicine status, intake and output status and patient's education $(100 \%)$; while the nursing report structure was regarded in all cases $(100 \%)$. The results showed that there is a significant difference in the quality of reporting before and after using CCC $(p<0.001)$.

Conclusions: Since the necessary parameters for recording a standard report do exist in electronic reporting (CCC), from one point, nurses are reminded to record the necessary parts and from the other point, the system does not allow the user to shut it down unless the necessary parameters are recorded. For this reason, the quality of recorded reports with electronic reporting improves.

Keywords: Nursing records; Nursing process; Electronic health record; Electronic medical records; Hospital
\end{abstract} information system

\section{Introduction}

Clinical care presentation is the main responsibility of the nurses, and writing nursing reports indicates these care tasks are done (1). Therefore, documentation is necessary in nursing (2). Recording of nursing reports includes the results of care and the patient's response, therefore nothing can indicate the appropriateness of presented services except a perfect standard document. An incomplete recording of the nursing report endangers the patient's safety and the nurse's position $(3,4)$. Despite the importance of documentation, research both inside and outside the country indicate the undesirable situation of care recordings and a lack of a suitable framework for recording nursing care (4, 5).The nurse's lack of time, carelessness in recording and manual recording of nursing reports in some cases, make it impossible to investigate actions which were done, due to illegibility of the recorded reports. In most cases, there is

\section{Corresponding author:}

Associate Professor Dr. Maryam Rassouli, School of Nursing \& Midwifery, Shahid Beheshti University of Medical Sciences, Tehran, Iran. Tel: +982188202519, Fax: +982188202521, Email: rassouli.m@gmail.com

Received: December 12, 2016, Accepted: May 20, 2017, Published: October 2017

iThenticate screening: May 18, 2017, English editing: July 03, 2017, Quality control: August 25, 2017

(C) 2017 The Authors. This is an open access article under the terms of the Creative Commons Attribution-NonCommercialNoDerivs License, which permits use and distribution in any medium, provided the original work is properly cited, the use is non-commercial and no modifications or adaptations are made. 
no legal defense of the nurse because of the gradual fading and illegibility of manual records (4-6). The results, taken out by the committee of deaths and medical errors, showed that there are 20 legal cases related to inaccurate recording of nursing reports from February to August 2016. Although all patient care tasks had been completed, the nurses were reprimanded and investigated by internal and external authorities due to inaccurate recording. Because of which, nurses were reprimanded by both the hospital's internal and external legal authorities despite having carried out all required actions for the patients. It's very difficult to retrieve data in Iran since most recording systems are manual and are similar to writing stories without a defined structure or framework (4-6). Nowadays, with the development of nursing care, the amount of its documents is increasing (7). Consequently, in order to manage this huge amount of data, the use of computers in health data recording is increasing among different countries $(2,8,9)$ and electronic reporting seems to be the best method of recording nursing reports $(10,11)$. This method which was used in the late 1980s, made it possible to record a large amount of patient's information in the nurse's electronic file, so the treatment team could access it easily $(8,11,12)$.Though, studies show that different countries have different structures for recording nursing reports; however, the most common pattern and structure for recording nursing reports is the nursing process (9). Among all standard nursing record computer systems, only Clinical Care Classification (CCC) and International Classification of Nursing Practice (ICNP) are capable of recording, encoding and classifying the interventions and nursing diagnosis $(8,14)$, and they can be applied in all nursing dimensions $(13,14)$. Unofficial studies of fourteen hospitals of Lorestan province, Iran, indicate that while nurses are familiar with standard nursing diagnosis, only two hospitals use them in recording nursing reports and others do not. Recording nursing reports plays a vital role in the coordination between treatment team members, continuity of care and nurses' partnership in professional duty and responsibilities, intervention's evaluation, determining nursing care expenses and protecting patients' and nurses' rights. Since recording taken actions in the nursing report framework is one of the main responsibilities of nurses, most nurses consider it as a routine and repetitive job and unfortunately, their care and sensitivity diminishes in recording standard nursing records over time. So, using electronic nursing reports is a good way to increase care in recording. Although, according to some studies, there is no evidence of the effect of the electronic recording system on the improvement of documentation quality $(2,11,15,16)$, some others consider it an effective factor in the improvement of care quality and nurses' recordings $(5,17)$. A 2014 study by Kahouei et al. showed that a hospital information system increases the quality of patient care (11). Other studies have also shown that using electronic recordings of nursing reports, directly affects the quality of reporting $(1,5)$. What counts to be investigated is considering the efficacy of utilizing different electronic reporting systems. Since the efficacy of every system has to be evaluated before utilizing, this study aims at determining the effectiveness of applying electronic recordings of nursing reports on the quality of nursing report recording based on CCC.

\section{Material and Methods}

This quasi-experimental study was conducted in the heart department of Shahid Rahimi Medical Center, Lorestan University of Medical Science from 2014 to 2015. The samples were nursing reports on the hospitalized patients in the heart department, the basis of complete enumeration (census) during the fall of 2014. The subjects were sixteen employed nurses. To do the study, the software of nursing records was design based on the Clinical Care Classification (CCC) system. The research's tool was the checklist of the Standards of Nursing Documentation. First, searching the databases including google scholars, science direct, Pub Med and web of science between the years 2000 and 2013, report writing standards which were noted in reliable resources of articles and books both in English and Persian. A checklist was designed containing 102 words in 13 main groups: admission, 11 parameters; examination results, 4 parameters; medications record, 6 parameters; adsorption and desorption record, 7 parameters; hemodynamic record, 6 parameters; activity record, sleep, rest, 4 parameters; acute changes, 8 parameters; record of the steps taken, 9 parameters; paraclinic record, 21 parameters; entry phone, 4 parameters; patient teaching, 2 parameters; the nursing process, 11 parameters; structure of reporting, 9 parameters and in Likert scale 4 ratings including full registration ( 2 points), incomplete registration ( 1 point), not registered ( 0 points) and not included. In order to determine validity and reliability of the research tool, the content validity method and calculating coefficient of agreement between observers, $(\mathrm{ICC}=0.97)$ and Cronbach's alpha $(\alpha=0.98)$ were used. To do this, two nurses (educational and clinical supervisor) who had equal knowledge about standards of nursing documentation, were given fifteen nursing reports and they were asked to evaluate the nursing reports using checklists. Then, the coefficient of agreement between observers was determined. The amount of adaption of recorded nursing reports by nurses with report writing standards before applying the electronic system of recording nursing reports, and during three next months after executing intervention was investigated. Analytical statistics were used to compare scores before and after intervention by SPSS 20 . The recorded nursing reports during three months of autumn were first investigated, and then diagnosis and nursing interventions were extracted. Finally, the results were compared with CCC. The findings were delivered to eleven members of the nursing faculty and nurses 
familiar with the nursing process on the basis of Delphi technique and through email. These people were asked to use Yes and No in order to confirm or deny the adaption of each phrase. Considering the return of ten checklists from the eleven that were sent (91\%), and the amount of phrases' adaption ( $94.6 \%$ for diagnostic phrases and 95.5 $\%$ for interventional phrases), there was no need for the second Delphi step. Eventually, those nursing diagnoses and interventions which were confirmed from the adaption view were used in CCC designing. After coordinating with the software designer, the main concepts of nursing care, diagnosis, implementation, recording nursing report rules, and importance and the reason of recording nursing reports were explained. Several meetings were held during the initial designing of the software (three months) to repair its defects, facilitate its use and increase its adaption with report writing standards. After designing the software and installing it, which lasted about three months, nurses were trained to use it. At the first training session, the main classes of the CCC system were taught to the nurses and they received a copy of the information. Then, during the second session, after listening to the nurses' viewpoints, the software was introduced to them and other aspects were taught. Training sessions were held with a week interval while nurses were able to ask their questions and eliminate their vagueness every day from the researcher. Therefore, during the time of software application, nurses' suggestions were received and the second and third versions of the software were designed. After the passing of three months and designing the third version of the software, using the first step's tools, the quality of the recorded nursing reports of previous three months (January, February, March) were confirmed by the research council of Shahid Beheshti University of Medical Science and an ethical code with the number of IR.SBMU.PHNM.1395.372 was received. Permission for access to the recorded nursing reports and using the software of recording data was also given. Finally, volunteer nurses who were eligible to take part in the research, filled the informed consent form.

\section{Results}

Checking 258 patients' records before applying the electronic system of recording nursing reports showed that 160 patients were women with the average age range of 63 years, the main reason of their hospitalization was related to chest pain and the average time of hospitalization was six days. However, checking 285 patient's records after applying the electronic system of recording nursing reports showed that 153 patients were women with the average age range of 62 years, while chronic heart failure was the main reason of hospitalization and the average time of hospitalization was seven days. The findings before and after running the CCC software were as follows: Before running the software, 450 nursing reports and after running it, 480 nursing reports were recorded which were investigated during three months. The findings indicated that from 13 phrases of the applied checklist related to the 450 recorded reports in the first step, included 5,850 phrases on the whole with 11,700 scores. Before the intervention, the whole score was 2,556 which included $16.8 \%$ for complete recording, $10.2 \%$ for incomplete recording, $49.5 \%$ for not recorded and finally, $23.5 \%$ for not included, while the amount of reports' adaption with the written standards was $21.8 \%$. The most complete recording was related to medicine status recording with the number of 261 cases $(58 \%)$ and the most not recorded were related to recording phone orders with the number of 450 cases $(100 \%)$, recording acute changes with the number of $446(99.1 \%)$ and recording nursing processes with the number of 351 cases (78\%). After interventions were done and CCC electronic reporting was used, the whole score was 8,343 from thirteen phrases, including complete recordings with $70.3 \%$, incomplete recording with 2 percent, not recorded with $4.4 \%$ and not included with $23.3 \%$, while the amount of reports' adaption with written standards was $71.3 \%$. The most complete recording was related to the medicine status recording, intake and output status recording and recording of patient's education with $100 \%$ (Table 1), while the nursing report structure was regarded in all cases $(100 \%)$ (Table 2$)$. Findings indicate a significant difference with $(p<0.001)$ and $(\alpha=0.5)$ before and after applying CCC (Table 3).

Table 1. The comparison of relative frequency of medicine recording, intake and output, activity and sleeping and patients' education in nursing reports with report writing standards before and after applying electronic reporting (CCC) in the heart department of Shahid Rahimi Medical Center from 2014 to 2015.

\begin{tabular}{|l|l|l|l|l|l|l|l|l|}
\hline \multirow{2}{*}{ Variables } & \multicolumn{2}{l|}{ Medicine recording } & \multicolumn{2}{l|}{ Intake and Output } & \multicolumn{2}{l|}{ Activity \& sleeping } & \multicolumn{2}{l|}{ Patient's education } \\
\cline { 2 - 10 } & Before & After & Before & After & Before & After & Before & After \\
\hline Complete recording & 58.4 & 100 & 31.1 & 100 & 30 & 100 & 0 & 100 \\
\hline Incomplete recording & 0 & 0 & 10.1 & 0 & 0.1 & 0 & 96.2 & 0 \\
\hline Not recorded & 41 & 0 & 57.3 & 0 & 68.9 & 0 & 3.8 & 0 \\
\hline Not included & 0.6 & 0 & 1.5 & 0 & 1 & 0 & 0 & 0 \\
\hline
\end{tabular}


http://www.ephysician.ir

Table 2. Absolute frequency and relative frequency of adaption of nursing report structure and nursing process recording status before and after applying electronic reporting (CCC) in the heart department of Shahid Rahimi Medical Center from 2014 to 2015.

\begin{tabular}{|l|l|l|l|l|}
\hline \multirow{2}{*}{ Variables } & \multicolumn{2}{|l|}{ Nursing report structure } & \multicolumn{2}{l|}{ Nursing process recording } \\
\cline { 2 - 5 } & Before & After & Before & After \\
\hline Complete recording & $169(38 \%)$ & $480(100 \%)$ & 0 & $400(83.3 \%)$ \\
\hline Incomplete recording & $112(24 \%)$ & 0 & $57(13 \%)$ & $35(7.3 \%)$ \\
\hline Not recorded & $169(38 \%)$ & 0 & $350(78 \%)$ & $45(9.4 \%)$ \\
\hline Not included & 0 & 0 & $43(9 \%)$ & 0 \\
\hline Sum total & $450(100 \%)$ & $480(100 \%)$ & $450(100 \%)$ & $480(100 \%)$ \\
\hline Test results & 24.450 & & 59.331 & \\
\hline P-value & $0.000^{*}$ & $0.000^{*}$ & \\
\hline
\end{tabular}

*significant at level $<0.001$

Table 3. The comparison between relative frequency of nursing report adaption and report writing standards, before and after applying CCC in the heart department of Shahid Rahimi Medical Center from 2014 to2015.

\begin{tabular}{|l|l|l|}
\hline The necessary principles of nursing report & Test results & $\mathrm{p}$-value \\
\hline Admission status & 2.907 & 0.034 \\
\hline Examination results & 3.472 & $0.007^{*}$ \\
\hline Medicine status & 17.828 & $0.00^{*}$ \\
\hline Intake and Output status & 24.799 & $0.00^{*}$ \\
\hline Hemodynamic status & 19.969 & $0.00^{*}$ \\
\hline Activity, sleeping and resting status & 31.807 & $0.000^{*}$ \\
\hline Acute changes & 33.004 & $0.00^{*}$ \\
\hline Intervention & 146.597 & $0.000^{*}$ \\
\hline Para clinical tests & 43.994 & $0.000^{*}$ \\
\hline Phone orders & 4.810 & $0.000^{*}$ \\
\hline Patient's education & 115.338 & $0.000^{*}$ \\
\hline
\end{tabular}

*significant at level $<0.01$

\section{Discussion}

The electronic nursing report is a method for recording nursing actions using standard nursing terminology which improves information quality, information sharing and decision making $(18,19)$. Since using this method seems to improve the quality of nursing reports, this study was done to investigate the effect of electronic report writing on the quality of nursing report recording. The findings indicated that the quality of recorded reports before the interventions done during this study was not in a good situation, which corresponds with previous studies' results in Iran $(1,5)$. Lack of implementation of nursing process in nursing report recording, lack of nurses, lack of time, irrelevant duty carried out by nurses, manual recording of nursing report and the type of department are factors affecting the quality of nursing report (1). Moreover, there was no cohesion in the reports despite the necessity to continue care in order to complete caring and treatment. Applying electronic reporting CCC fixes this problem, (1, $5,8)$. The results of surveys in the first step pointed out that the highest quality of nursing report was related to medicine recordings while recording phone orders revealed the lowest quality (20). However, in some other cases where the medicine and intake and output recording gained the highest quality score, sleeping and hemodynamic status recording were reported undesirable. Distribution of medicine is one the main responsibilities of nurses, which is naturally vital. Its compensations and legal consequences are fateful and can create numerous legal problems for nurses (21-23). Therefore, nurses are very careful in recording medicines and regarding standards since the task is repeated during all shifts and evaluated by physicians during their visits and during every monitoring. The results showed that since patients' medication is one of the most costly elements to consider, inaccurate recording produces a great deal of expenditure for the patient; on the other hand, monitoring systems in hospitals are sensitive to the exact registration. Nurses are also sensitive to cases with high financial burden and take more heed. Therefore, nurses are more sensitive to record items with financial results because they will be evaluated by surveillance systems $(24,25)$. Thus, the record of medication requires higher quality $(18,24,25)$. The data acquired after applying the electronic system of nursing report in the field of medicine recording, points out that the existence of a standard framework for medicine recording in CCC and commitment for careful recording of its entire items, made the medicine the most complete recorded item. "it plays an error" While it has a reminder for items needed to be 
recorded, it displays a flaw in it not recording all items to complete recording.. Undesirability of phone medicine order recordings, the status of output, sleeping and resting, activities and acute changes $(4,20,26)$ were confirmed in a number of studies. Applying the software has improved the quality of medicine, intake and output status, activity, sleeping and resting and patient's education. The status of electronic recorded reports can be proved with the improvement of the status of recording parameters relevant to hemodynamic status, acute changes recording and para clinical items recording. While some studies confirm the results $(1,20)$ some others suggest more research on the subject and carrying out comparative studies which are inconsistent to the results of this study (19). Despite using the nursing process as a part of recorded nursing reports in the studied department, the recorded nursing process did not meet the required standards since the recording pattern was not structuralized, and its most important problem was the lack of relevance between the recorded nursing diagnosis with the patient's problem. In electronic nursing reporting (CCC), it is necessary to be aware of a patient's status to select the correct nursing diagnosis because the diagnosis recording path to evaluation step includes several other steps and it is selected based on the patient's situation. From a structure point of view, CCC is one of the standard methods of recording nursing reports and improves the quality of recorded nursing reports. The results of other studies also confirm this $(8,14)$. As it has been stated in nursing records, prior to utilizing the software, no parameters related to nursery process have been mentioned in nursing reports. Since this is one of the most important factors in the assessment of wards and personnel, utilizing electronic recording not only will increase the quality of recording, but will also improve the hospital's accreditation. Lack of nurses, nurse's relatedness with the patient and replacement of department nurses were limitations of the study; however, the number of nurse's replacements decreased to its minimum after some negotiations. The findings of this study can be applied to improve the quality of nursing reports and consequently, improve nursing care. Further research can be done on applying the designed software in other departments, especially in critical care units as research projects.

\section{Conclusions}

In electronic reporting (CCC), the necessary parameters for recording a standard report does exist in this system. Also recording these parameters gives reminders to nurses, and in the case of not recording the necessary parameters, completing the report and closing the system is not allowed. For this reason, the quality of recorded reports with electronic reporting CCC is better than manual ones. Hence, it seems that utilizing electronic recording will improve the quality of nursing recording.

\section{Acknowledgments:}

This study is a part of a research design, approved by Shahid Beheshti University of Medical Science research council, and also a part of a nursing PhD thesis. This project consumed huge amounts of work, research and dedication. Still, implementation would not have been possible if we did not have the support of many individuals and organizations. Therefore we would like to extend our sincere gratitude to all of them. No fund or budget was assigned to this study.

\section{Conflict of Interest:}

There is no conflict of interest to be declared.

Authors' contributions:

All authors contributed to this project and article equally. All authors read and approved the final manuscript.

\section{References:}

1) Toolabi T, Vanaki Z, Memarian R, Namdari M. Quality of nursing documentations in CCU by hospital information system (HIS). Journal of Critical Care Nursing. 2012; 5(2): 53-62.

2) Meißner A, Schnepp W. Staff experiences within the implementation of computer-based nursing records in residential aged care facilities: a systematic review and synthesis of qualitative research. BMC Med Inform Decis Mak. 2014; 14: 54. doi: 10.1186/1472-6947-14-54. PMID: 24947420 , PMCID: PMC4114165.

3) Varzeshnezhad M, Rassouli M, Zaghari Tafreshi M, Kashef Ghorbanpour R, Moss J. Transcultural mappingand usability testing of the clinical care classification system for an Iranian neonatal ICU population. Comput Inform Nurs. 2014; 32(4): 182-8. doi: 10.1097/CIN.0000000000000032. PMID: 24429835 . 
4) Jasemi M, Zamanzadeh V, Rahmani A, Mohajjel A, Alsadathoseini F. Knowledge and Practice of Tabriz Teaching Hospitals' Nurses Regarding Nursing Documentation. Thrita. 2013; 2(2): 133-8. doi: 10.5812/thrita.8023.

5) Nasiriani K, Dehqani H, Akbari Roknabadi M. Nursing documentation requirements in coronary care unit. Critical Care Nursing. 2014; 7(3): 132-41.

6) Ahmadi M, Habibi Koolaee M. Nursing Information Systems in Iran. Hakim Research Journal. 2010; 13(3): 185-91.

7) Cherry BJ, Ford EW, Peterson LT. Experiences with electronic health records: Early adopters in long-term care facilities. Health Care Manage Rev. 2011; 36(3): 265-74. doi: 10.1097/HMR.0b013e31820e110f. PMID: 21646885.

8) Saranto K, Kinnunen UM, Kivekäs E, Lappalainen AM, Liljamo P, Rajalahti E, et al. Impacts of structuring nursing records: a systematic review. Scand J Caring Sci. 2014; 28; 629-47. doi: 10.1111/scs.12094. PMID: 24245661.

9) Dal Sasso GTM, Barra DCC, Paese F, Almeida SRWD, Rios GC, Marinho MM, Debetio MG. Computerized nursing process: methodology to establish associations between clinical assessment, diagnosis, interventions, and outcomes. Rev Esc Enferm USP. 2013; 47(1): 238-45

10) Johnson M, Jefferies $D$, Nicholls $D$. Developing a minimum data set for electronic nursing handover. J Clin Nurs. 2012; 21(3 - 4): 331-43. doi: 10.1111/j.1365-2702.2011.03891.x. PMID: 22082347.

11) Kahouei M, Baba Mohammadi H, Askari Majdabadi H, Solhi M, Parsania Z, Said Roghani P, et al. Nursing Information System: a Module of Electronic Medical Record for Patient Care in Two University Hospitals of Iran. Meter Sociomed. 2014;26(1):30-4. PMID: 24757398, PMCID: PMC3990381.

12) Min YH, Park H, Chung E, Lee H. Implementation of a next-generation electronic nursing records system based on detailed clinical models and integration of clinical practice guidelines. Healthc Inform Res. 2013; 19(4): 301-6. doi: 10.4258/hir.2013.19.4.301. PMID: 24523995, PMCID: PMC3920043.

13) Ahmadi M, Rafii F, Hoseini F, Habibi Koolaee M. A Comparison of Nursing Data Classification Systems. Health Information Management. 2012; 8(6): 852-60.

14) Moss J, Saba V. Costing Nursing Care Using the Clinical Care Classification System to Value Nursing Intervention in an Acute-Care Setting. Comput Inform Nurs. 2011; 29(8): 455-60. doi: 10.1097/NCN.0b013e3181 fcbe55. PMID: 21084972.

15) Ahmadi M, Rafii F, Hoseini F, Habibi Koolaee M, Mirkarimi A. Informational and Structural Needs of Nursing Data Classification in Computerized Systems. Hayat. 2011; 1: 16-23.

16) Wang N, Hailey D, Yu P. Quality of nursing documentation and approaches to its evaluation: a mixed method systematic review. J Adv Nurs. 2011; 67(9): 1858-75. doi: 10.1111/j.1365-2648.2011.05634.x. PMID: 21466578.

17) Jefferies $D$, Johnson $M$, Griffiths R. A meta - study of the essentials of quality nursing documentation. Int J Nurs Pract. 2010; 16(2): 112-24. doi: 10.1111/j.1440-172X.2009.01815.x. PMID: 20487056.

18) Park H, Min YH, Jeon E, Chung E. Integration of evidence into a detailed clinical model-based electronic nursing record system. Healthc Inform Res. 2012; 18(2): 136-44. doi: 10.4258/hir.2012.18.2.136. PMID: 22844649, PMCID: PMC3402556.

19) Kelley TF, Brandon DH, Docherty SL. Electronic nursing documentation as a strategy to improve quality of patient care. J Nurs Scholarsh. 2011; 43(2): 154-62. doi: 10.1111/j.1547-5069.2011.01397.x. PMID: 21605319.

20) Mohamad Ghasaby M, Masudi Alavi N. Quality and barriers against nursing documentation in Kashan Shahid Beheshti Hospital (2011). Mod Care J. 2013; 9(4): 336-43.

21) Alijanzadeh M, Mohebi Far R, Azadmanesh Y, Faraji M. The Frequency of Medication Errors and Factors Influencing the Lack of Reporting Medication Errors in Nursing at Teaching Hospital of Qazvin University of Medical Sciences, 2012. Journal of Health. 2015; 6(2): 169-79.

22) Azizi-Fini I, Adib-Hajbaghery M. Nursing Assistants, Drug Medication Errors, and Patient Safety: A New Challenge in Iran. Nurs Midwifery Stud. 2016; 4(3): e34273. doi: 10.17795/nmsjournal34273. PMID: 27331061, PMCID: PMC4915206.

23) Soltanian M, Molazem Z, Mohammadi E, Sharif F, Rakhshan M. Iranian Nurses' Experiences on Obstacles of Safe Drug Administration: A Qualitative Study. Glob J Health Sci. 2016; 8(10): 56009. doi: 10.5539/gjhs.v8n10p88. PMID: 27302450. 
24) Khorrmmy F, Eshpala RH, Baniasadi T, Azarmehr N, Mohammady F. Prioritizing insurance deductions factors of Shahid Mohammadi hospital inpatients records using Shannon Entropy, Bandar Abbas, Iran. Medical Journal of Hormozgan University. 2013; 17(1): 77-82.

25) Moalemi S, Shams Abadi AR, Meshkani Z, Alikiani A, Kazemi Karyani A. Survey and comparison on the causes of deduction in admitted social insurance: Bahonar and Arjmand hospital in Kerman. Iran Health Information Management Association. 2014; 8(1): 17-24.

26) Saif Rabiei MA, Sedighi I, Mazdeh M, Dadras F, Shokouhee Solgi M, Moradi A. Study of hospital records registration in teaching hospital of Hamadan University of Medical Sciences in 2009. Scientific Journal of Hamadan University of Medical Sciences. 2010; 16(2): 45-9. 